\title{
Multiple Solution Results for Perturbed Fractional Differential Equations with Impulses
}

\author{
Peiluan Li $\mathbb{D},{ }^{1}$ Liang Xu $\mathbb{D},{ }^{1}$ Peiyu Li $\mathbb{D},{ }^{2}$ and Hui Wang ${ }^{3}$ \\ ${ }^{1}$ School of Mathematics and Statistics, Henan University of Science and Technology, Luoyang 471023, China \\ ${ }^{2}$ College of Information Engineering, Henan University of Science and Technology, Luoyang 471023, China \\ ${ }^{3}$ Network Information Center, Henan University of Science and Technology, Luoyang 471023, China
}

Correspondence should be addressed to Peiluan Li; lpllpl_lpl@163.com

Received 31 July 2020; Revised 10 October 2020; Accepted 15 October 2020; Published 12 November 2020

Academic Editor: Fanglei Wang

Copyright (C) 2020 Peiluan Li et al. This is an open access article distributed under the Creative Commons Attribution License, which permits unrestricted use, distribution, and reproduction in any medium, provided the original work is properly cited.

The multiplicity of classical solutions for impulsive fractional differential equations has been studied by many scholars. Using Morse theory, Brezis and Nirenberg's Linking Theorem, and Clark theorem, we aim to solve this kind of problems. By this way, we obtain the existence of at least three classical solutions and $k$ distinct pairs of classical solutions. Finally, an example is presented to illustrate the feasibility of the main results in this paper.

\section{Introduction}

Consider the multiple solutions of fractional order impulsive systems as follows:

$$
\left\{\begin{array}{l}
-\frac{1}{2} \frac{d}{d t}\left({ }_{0} D_{t}^{-\beta}+{ }_{t} D_{T}^{-\beta}\right) u^{\prime}(t)=a(t) u(t)+\nabla F(t, u(t)), \quad t \neq t_{j}, \text { a.e. } t \in[0, T] \\
\Delta\left(D_{t}^{\alpha} u\right)\left(t_{j}\right)=I_{j}\left(u\left(t_{j}\right)\right), \quad t_{j} \in[0, T], j=1,2, \cdots, l, \\
u(0)=u(T)=0
\end{array}\right.
$$

where $\beta \in[0,1), \alpha=1-\beta / 2 \in(1 / 2,1] ;{ }_{0} D_{t}^{-\beta},{ }_{t} D_{T}^{-\beta}$ are the left and right Riemann-Liouville fractional integrals of order $\beta$, ${ }_{0}^{c} D_{t}^{\alpha}{ }_{t}^{c} D_{T}^{\alpha}$ are used to denote the left and right Caputo fractional derivatives of order $\alpha, 0=t_{0}<t_{1}<\cdots<t_{l}<t_{l+1}=T, a$ $\in C[0, T], F:[0, T] \times R^{N} \longrightarrow R$ is a given function, $\nabla F(t, x)$ is the gradient of $F$ at $x$, there are constants $a_{0}, a_{1}$ with $0<$ $a_{0} \leq a(t) \leq a_{1}$,

$$
\left(D_{t}^{\alpha} u\right)(t)=\frac{1}{2}\left\{{ }_{0} D_{t}^{\alpha-1}\left({ }_{0}^{c} D_{t}^{\alpha} u\right)-{ }_{t} D_{T}^{\alpha-1}\left({ }_{t}^{c} D_{T}^{\alpha} u\right)\right\}(t)
$$

$$
\begin{aligned}
& \Delta\left(D_{t}^{\alpha} u\right)\left(t_{j}\right)= \frac{1}{2}\left\{{ }_{0} D_{t}^{\alpha-1}\left({ }_{0}^{c} D_{t}^{\alpha} u\right)-{ }_{t} D_{T}^{\alpha-1}\left({ }_{t}^{c} D_{T}^{\alpha} u\right)\right\}\left(t_{j}^{+}\right) \\
&-\frac{1}{2}\left\{{ }_{0} D_{t}^{\alpha-1}\left({ }_{0}^{c} D_{t}^{\alpha} u\right)-{ }_{t} D_{T}^{\alpha-1}\left({ }_{t}^{c} D_{T}^{\alpha} u\right)\right\}\left(t_{j}^{-}\right), \\
&\left\{{ }_{0} D_{t}^{\alpha-1}\left({ }_{0}^{c} D_{t}^{\alpha} u\right)-{ }_{t} D_{T}^{\alpha-1}\left({ }_{t}^{c} D_{T}^{\alpha} u\right)\right\}\left(t_{j}^{+}\right) \\
&=\lim _{t \rightarrow t_{j}^{+}}\left\{{ }_{0} D_{t}^{\alpha-1}\left({ }_{0}^{c} D_{t}^{\alpha} u\right)-{ }_{t} D_{T}^{\alpha-1}\left({ }_{t}^{c} D_{T}^{\alpha} u\right)\right\}(t), \\
&\left\{{ }_{0} D_{t}^{\alpha-1}\left({ }_{0}^{c} D_{t}^{\alpha} u\right)-{ }_{t} D_{T}^{\alpha-1}\left({ }_{t}^{c} D_{T}^{\alpha} u\right)\right\}\left(t_{j}^{-}\right) \\
&=\lim _{t \rightarrow t_{j}^{-}}\left\{{ }_{0} D_{t}^{\alpha-1}\left({ }_{0}^{c} D_{t}^{\alpha} u\right)-{ }_{t} D_{T}^{\alpha-1}\left({ }_{t}^{c} D_{T}^{\alpha} u\right)\right\}(t),
\end{aligned}
$$

for $j=1,2, \cdots, l$.

The problem (1) arises from the phenomena of advection dispersion and was first scrutinized by Erwin and Roop in [1]. From then on, more and more scholars began to pay attention to the problem in [1] and the related problems.

Fractional calculus is different from integral calculus in nature. It has nonlocal characteristics and is very suitable for describing materials and processes with memory effect and genetic properties. Therefore, fractional differential equations are widely used in many domains, for instance, 
biomedicine, economic mathematics, and technology science $[2,3]$. In recent years, the variational methods and critical point theory have been widely used to study fractional differential equations [4-8].

In [8], the authors discussed the following fractional order differential systems:

$$
\left\{\begin{array}{l}
-\frac{1}{2} \frac{d}{d t}\left({ }_{0} D_{t}^{-\beta}+{ }_{t} D_{T}^{-\beta}\right) u^{\prime}(t)=\nabla F(t, u(t)), \quad \text { a.e. } t \in[0, T] \\
u(0)=u(T)=0 .
\end{array}\right.
$$

They used the critical point theory and other tools to verify the existence of solutions. From then on, a number of scholars began to use such methods for research, as shown in [9-11].

In [12], the authors discussed the following problems:

$$
\left\{\begin{array}{l}
{ }_{t} D_{T}^{a}\left(a(t){ }_{0} D_{t}^{\alpha} u(x)\right)=\lambda u(t) \nabla F(t, u(t)), \quad \text { a.e. } t \in[0, T] \\
u(0)=u(T)=0
\end{array}\right.
$$

They proved that there are at least $k$ pairs of weak solutions and two weak solutions by using the Clark Theorem and other methods.

An impulsive phenomenon is a common phenomenon in nature and engineering applications. The models reflected in mathematics are impulsive differential equations. The most prominent feature of impulsive differential equation is that it can fully consider the impact of instantaneous mutation on the state. Therefore, in recent decades, impulsive differential equation theory has been widely used in biological mathematics, theoretical mechanics, biomedicine, and economic mathematics (see [13-18]).

For the past few years, very few scholars used the variational method and critical point theory to discuss impulsive fractional differential equations and their boundary value problems. Moreover, few papers discuss the fractional order system by using Morse theory (see [19-24]).

In [23], the authors discussed the following problems:

$$
\left\{\begin{array}{l}
{ }_{t} D_{T}^{\alpha}\left({ }_{0}^{c} D_{t}^{\alpha} u(t)\right)+k(t) u(t)=f(t, u(t)), \quad 0<t<T, t \neq t_{j}, \\
\Delta\left({ }_{t} D_{T}^{\alpha-1}\left({ }_{0}^{c} D_{t}^{\alpha_{i}} u\right)\right)\left(t_{j}\right)=I_{j}\left(u\left(t_{j}\right)\right), \quad j=1,2, \cdots, m, \\
u(0)=u(T)=0 .
\end{array}\right.
$$

The multiple solutions of this problem are verified with Morse theory and the Clark theorem by the authors.

In [25], the sufficient conditions for the existence of infinite solutions to the system (1) are obtained by using the variational method.

Based on the above literatures, in the present paper, we will discuss the existence of multiple classical solutions for (1) by using Morse theory, Clark theorem, and Brezis and Nirenberg's Linking Theorem.

First of all, we give some assumptions.

$(H 1) I_{j} \in C([0, T], R), I_{j}(0)=0$, there exist some constants $e_{j}, \gamma_{j} \in[0,1), a_{j}, b_{j}>0$, such that $\lim _{|u| \rightarrow 0}\left(\left|I_{j}(u)\right| /|u|^{e_{j}}\right)=b_{j}, \mid I_{j}$ $\left.(u)\left|\leq a_{j}\right| u\right|^{\gamma_{j}}, j=1, \cdots, l$, and $\int_{0}^{u} I_{j}(s) d s \geq 0$, for $\forall u \in R$

$(H 2) F \in C([0, T], R)$ and $F(t, 0)=0, \lim _{|u| \rightarrow \infty} \sup (F(t, u)$ $\left./|u|^{2}\right)<1 / 2\left(\Gamma^{2}(\alpha+1)\left|\cos (\pi \alpha) / T^{2 \alpha}\right|-a_{1}\right)$ uniformly on $t$ $\in[0, T]$

(H3) $\lim _{|u| \rightarrow 0} \sup \left(|F(t, u)| /|u|^{2}\right)<\Gamma^{2}(\alpha+1) / 2 T^{2 \alpha}$ uniformly for $t \in[0, T]$. There exist four constants $C>0, r, r_{0}, \gamma \in(1$, $\left.\max _{j \in 1,2, \cdots, l}\left\{\gamma_{j}+1\right\}\right)$ such that $F(t, u) \geq C|u|^{\gamma}, r \leq|u| \leq r_{0}$, a.e.t $\in[0, T]$

$(H 4) F(t,-u)=F(t, u)$ and $I_{j}(-u)=-I_{j}(u)(j=1,2, \cdots, l)$, for $u \in R$

The key outcomes are as follows.

Theorem 1. Let (H1)-(H3) hold. Then, the problem (1) has at least three classical solutions.

Theorem 2. Let (H1)-(H4) hold. Then, the problem (1) has at least $k$ distinct pairs of classical solutions.

Note that the methods in this article are distinct from [25] and our results are richer. The problems in this paper we studied are different from the problems in [23]. Compared with [23], classical solutions are investigated in this paper.

The structure of this article is as below. In Section 2, we provide some preliminary knowledge, which are helpful to the proof the key outcomes. We prove the key outcomes in Section 3. Finally, an example is given to illustrate the main results.

\section{Preliminaries}

Similar to [25], we first convert system (1) into a new format as follows:

$$
\left\{\begin{array}{l}
\frac{d}{d t}\left\{\frac{1}{2}{ }_{0} D_{t}^{\alpha-1}\left({ }_{0}^{c} D_{t}^{\alpha} u(t)\right)-\frac{1}{2}{ }_{t} D_{T}^{\alpha-1}\left({ }_{t}^{c} D_{T}^{\alpha} u(t)\right)\right\}+a(t) u(t)+\nabla F(t, u(t))=0, \quad t \neq t_{j}, \text { a.e.t } \in[0, T], \\
\Delta\left(D_{t}^{\alpha} u\right)\left(t_{j}\right)=I_{j}\left(u\left(t_{j}\right)\right), \quad t_{j} \in(0, T), j=1,2, \cdots, l, \\
u(0)=u(T)=0 .
\end{array}\right.
$$


Remark 3. Because of the equivalence of system (1) and system (6), we know that the solutions of system (6) are the solutions of system (1).

We first build the function spaces as below, the goal of which is to establish the variational framework of system (6).

Let us recall that for any fixed $1 \leq p \leq \infty$ and $t \in[0, T]$, $\|u\|_{\infty}=\max _{t \in[0, T]}|u(t)|,\|u\|_{L^{p}}=\left(\int_{0}^{T}|u(s)|^{p} d s\right)^{1 / p}$.

Definition 4 (see [22]). Let $0<\alpha \leq 1$, we define the fractional derivative space $E_{0}^{\alpha}$ by the closure of $C_{0}^{\infty}([0, T], R)$ with $u(0)$ $=u(T)$ under the norm

$$
\|u\|_{\alpha}=\left(\int_{0}^{T}\left|{ }_{0}^{c} D_{t}^{\alpha} u(t)\right|^{2} d t+\int_{0}^{T}|u(t)|^{2} d t\right)^{1 / 2}, \quad \forall u \in E_{0}^{\alpha}
$$

Lemma 5 (see [25]). Let $0<\alpha \leq 1$, and $E_{0}^{\alpha}$ is a Banach space with reflexive and separable.

Definition 6 (see [25]). We define that the function $u \in E_{0}^{\alpha}$ is a weak solution of the system (6) if the following holds:

$$
\begin{gathered}
\int_{0}^{T}\left\{-\frac{1}{2}\left[{ }_{0}^{c} D_{t}^{\alpha} u(t){ }_{t}^{c} D_{T}^{\alpha} v(t)+{ }_{t}^{c} D_{T}^{\alpha} u(t){ }_{0}^{c} D_{t}^{\alpha} v(t)\right]-a(t) u(t) v(t)\right\} d t \\
+\sum_{j=1}^{l} I_{j}\left(u\left(t_{j}\right)\right) v\left(t_{j}\right)-\int_{0}^{T} \nabla F(t, u(t)) v(t) d t=0
\end{gathered}
$$

for $\forall v \in E_{0}^{\alpha}$.

We define $\Phi: E_{0}^{\alpha} \longrightarrow R$ as

$$
\begin{aligned}
\Phi(u)= & \int_{0}^{T}-\frac{1}{2}{ }_{0}^{c} D_{t}^{\alpha} u(t){ }_{t}^{c} D_{T}^{\alpha} u(t) d t-\frac{1}{2} \int_{0}^{T} a(t) u^{2}(t) d t \\
& -\int_{0}^{T} F(t, u(t)) d t+\sum_{j=1}^{l} \int_{0}^{u\left(t_{j}\right)} I_{j}(s) d s .
\end{aligned}
$$

From $(H 1),(H 2)$, we know the functional $\Phi$ is continuously differentiable. So $\forall u, v \in E_{0}^{\alpha}$, we have

$$
\begin{aligned}
\left\langle\Phi^{\prime}(u), v\right\rangle= & \int_{0}^{T}-\frac{1}{2}\left[{ }_{0}^{c} D_{t}^{\alpha} u(t){ }_{t}^{c} D_{T}^{\alpha} v(t)+{ }_{t}^{c} D_{T}^{\alpha} u(t){ }_{0}^{c} D_{t}^{\alpha} v(t)\right] d t \\
& -\int_{0}^{T} a(t) u(t) v(t) d t+\sum_{j=1}^{l} I_{j}\left(u\left(t_{j}\right)\right) v\left(t_{j}\right) \\
& -\int_{0}^{T} \nabla F(t, u(t)) v(t) d t .
\end{aligned}
$$

Remark 7. Obviously, from (10), we know that the critical points of functional $\Phi$ are the weak solutions of system (6).
Definition 8 (see [25]). We define

$u \in\left\{u \in A C([0, T]): \int_{t_{j}}^{t_{j+1}}\left(\int_{0}^{T}\left|{ }_{0}^{c} D_{t}^{\alpha} u(t)\right|^{2}+\int_{0}^{T}|u(t)|^{2}\right)^{1 / 2} d t<\infty, j=0, \cdots, l\right\}$,

as a classic solution of system (6) if it satisfies the following conditions:

(i) $1 / 2\left\{{ }_{0} D_{t}^{\alpha-1}\left({ }_{0}^{c} D_{t}^{\alpha} u\right)-{ }_{t} D_{T}^{\alpha-1}\left({ }_{t}^{c} D_{T}^{\alpha} u\right)\right\}\left(t_{j}^{+}\right)=\lim _{t \rightarrow t_{j}^{+}} 1 / 2\{$

$$
\begin{gathered}
\left.{ }_{0} D_{t}^{\alpha-1}\left({ }_{0}^{c} D_{t}^{\alpha} u\right)-{ }_{t} D_{T}^{\alpha-1}\left({ }_{t}^{c} D_{T}^{\alpha} u\right)\right\}(t), \\
\frac{1}{2}\left\{{ }_{0} D_{t}^{\alpha-1}\left({ }_{0}^{c} D_{t}^{\alpha} u\right)-{ }_{t} D_{T}^{\alpha-1}\left({ }_{t}^{c} D_{T}^{\alpha} u\right)\right\}\left(t_{j}^{-}\right) \\
=\lim _{t \rightarrow t_{j}^{-}} \frac{1}{2}\left\{{ }_{0} D_{t}^{\alpha-1}\left({ }_{0}^{c} D_{t}^{\alpha} u\right)-{ }_{t} D_{T}^{\alpha-1}\left({ }_{t}^{c} D_{T}^{\alpha} u\right)\right\}(t), \\
\Delta\left(D_{t}^{\alpha} u\right)\left(t_{j}\right)=I_{j}\left(u\left(t_{j}\right)\right), \\
u(0)=u(T)=0
\end{gathered}
$$

(ii) $u$ content system (1) a.e. on $t \in[0, T] \backslash\left\{t_{1}, t_{2}, \cdots, t_{l}\right\}$

Lemma 9 (see [25]). The function $u \in E_{0}^{\alpha}$ is a classical solution of system (6) when $u$ is a weak solution of system (6).

Remark 10. Combine Remarks 3 and 7 and Lemma 9, we know that the critical point of functional $\Phi$ is the classical solution of the system (1). Therefore, we will directly discuss the critical point of $\Phi$ as below.

Lemma 11 (see [8]). Let $1 / 2<\alpha \leq 1$ and $1<p<\infty$, for all $u$ $\in E_{0}^{\alpha}$, one has

$$
\|u\|_{L^{p}} \leq \frac{T^{\alpha}}{\Gamma(\alpha+1)}\left\|{ }_{0}^{c} D_{t}^{\alpha} u\right\|_{L^{p}}
$$

Moreover, if $\alpha>1 / p, 1 / p+1 / q=1$, then

$$
\|u\|_{\infty} \leq \frac{T^{\alpha-1 / p}}{\Gamma(\alpha)((\alpha-1) q+1)^{1 / q}}\left\|{ }_{0}^{c} D_{t}^{\alpha} u\right\|_{L^{p}}
$$

In particular, if $p=2$, then

$$
\begin{aligned}
& \|u\|_{L^{2}}^{2} \leq \frac{T^{2 \alpha}}{\Gamma^{2}(\alpha+1)}\|u\|_{\alpha}^{2}, \\
& \|u\|_{\infty}^{2} \leq \frac{T^{2 \alpha-1}}{\Gamma^{2}(\alpha)(2 \alpha-1)}\|u\|_{\alpha}^{2} .
\end{aligned}
$$

It is easy to prove that the norm $\|u\|_{\alpha}=$ $\left(\int_{0}^{T}\left|{ }_{0}^{c} D_{t}^{\alpha} u(t)\right|^{2} d t+\int_{0}^{T}|u(t)|^{2} d t\right)^{1 / 2}$ is equivalent to $\|u\|_{\alpha}=$ 
$\left(\int_{0}^{T}\left|{ }_{0}^{c} D_{t}^{\alpha} u(t)\right|^{2} d t\right)^{1 / 2}, \forall u \in E_{0}^{\alpha}$. Next, we will use $\|u\|_{\alpha}=$ $\left(\int_{0}^{T}\left|{ }_{0}^{c} D_{t}^{\alpha} u(t)\right|^{2} d t\right)^{1 / 2}$ as the norm in $E_{0}^{\alpha}$.

Lemma 12 (see [8]). Let $1 / 2<\alpha \leq 1, \forall u \in E_{0}^{\alpha}$, have

$$
|\cos (\pi \alpha)|\|u\|_{\alpha}^{2} \leq-\int_{0}^{T}{ }_{0}^{c} D_{t}^{\alpha} u(t){ }_{t}^{c} D_{T}^{\alpha} u(t) d t \leq \frac{1}{|\cos (\pi \alpha)|}\|u\|_{\alpha}^{2}
$$

Lemma 13 (see [8]). Let $1 / 2<\alpha \leq 1$. Assume the sequence $\left\{u_{n}\right\}$ converges weakly to $u$ in $E_{0}^{\alpha}$. Then, $u_{n} \longrightarrow u$ strongly in $C([0, T], R)$, i.e., $\left\|u_{n}-u\right\|_{\infty} \longrightarrow 0$, as $n \longrightarrow \infty$.

Definition 14 (see [23]). We say that $\Phi$ satisfies the (PS) condition in $E_{0}^{\alpha}$, if any $\left\{u_{n}\right\}_{n \in N} \subset E_{0}^{\alpha}$, for which $\left\{\Phi\left(u_{n}\right)\right\}_{n \in N}$ is bounded and $\Phi^{\prime}\left(u_{n}\right) \longrightarrow 0$ as $n \longrightarrow \infty$ owns a strongly convergent subsequence in $E_{0}^{\alpha}$.

Lemma 15 (see [26]). Let $E$ have a direct sum decomposition $E=V \oplus W$, and $k=\operatorname{dim} V<\infty$. Let 0 be a critical point of $\Phi$ with $\Phi(0)=0, \Phi$ is bounded below and satisfying (PS) condition. Suppose that, for some $\rho>0$,

$$
\begin{gathered}
\Phi(u) \leq 0, \quad \forall u \in V,\|u\| \leq \rho \\
\Phi(u)>0, \quad \forall u \in W,\|u\| \leq \rho .
\end{gathered}
$$

Also, assume that $\inf _{E} \Phi<0$. Then, $\Phi$ has at least two nonzero critical points and $C_{k}(\Phi, 0) \cong 0$.

Lemma 16 (see [27]). Let $E$ be a real Banach space, $\Phi \in$ $C^{l}(E, R)$; assume that $\Phi$ is even, bounded from below, and satisfying $(P S)$ condition. Assume $\Phi(0)=0$, there exists a set $E^{\prime} \subset E$ such that $E^{\prime}$ is homeomorphic to $S^{k-1}$ by an odd map, and $\sup \Phi<0$. Then, $\Phi$ has at least $k$ distinct pairs of critical points.

\section{Proofs of Main Results}

Lemma 17. Suppose (H1), (H2) hold, if $\left\{u_{n}\right\}$ is a (PS) sequence, then $\left\{u_{n}\right\}$ is bounded.

Proof. If $\left\{u_{n}\right\}$ is a $(P S)$ sequence, that is,

$$
\Phi\left(u_{n}\right) \text { is bounded, } \Phi^{\prime}\left(u_{n}\right) \longrightarrow 0 \text {, as } n \longrightarrow \infty \text {. }
$$

From (H2), for some $\xi>0$ small enough, there is a constant $C_{\xi}>0$, for any $u \in R, t \in[0, T]$ such that

$$
|F(t, u)| \leq \frac{1}{2}\left(\frac{\Gamma^{2}(\alpha+1)}{T^{2 \alpha}}|\cos (\pi \alpha)|-a_{1}-\xi\right)|u|^{2}+C_{\xi}
$$

According to (19), for $u \in E_{0}^{\alpha}, u \neq 0$, one has

$$
\begin{aligned}
\Phi(u)= & \int_{0}^{T}-\frac{1}{2}{ }_{0}^{c} D_{t}^{\alpha} u(t){ }_{t}^{c} D_{T}^{\alpha} u(t) d t-\frac{1}{2} \int_{0}^{T} a(t) u^{2}(t) d t \\
& -\int_{0}^{T} F(t, u(t)) d t+\sum_{j=1}^{l} \int_{0}^{u\left(t_{j}\right)} I_{j}(s) d s \geq \frac{1}{2}|\cos (\pi \alpha)|\|u\|_{\alpha}^{2} \\
& -\frac{1}{2}\left(\frac{\Gamma^{2}(\alpha+1)}{T^{2 \alpha}}|\cos (\pi \alpha)|-a_{1}-\xi\right) \int_{0}^{T}|u(t)|^{2} d t \\
& -\frac{a_{1}}{2} \int_{0}^{T} u^{2}(t) d t-C_{\xi} T=\frac{1}{2}|\cos (\pi \alpha)|\|u\|_{\alpha}^{2} \\
& -\frac{1}{2}\left(\frac{\Gamma^{2}(\alpha+1)}{T^{2 \alpha}}|\cos (\pi \alpha)|-\xi\right)\|u\|_{L^{2}}^{2}-C_{\xi} T \\
\geq & \frac{1}{2}|\cos (\pi \alpha)|\|u\|_{\alpha}^{2}-\frac{1}{2}\left(|\cos (\pi \alpha)|-\frac{T^{2 \alpha}}{\Gamma^{2}(\alpha+1)} \xi\right)\|u\|_{\alpha}^{2} \\
& -C_{\xi} T \geq \frac{\xi T^{2 \alpha}}{2 \Gamma^{2}(\alpha+1)}\|u\|_{\alpha}^{2}-C_{\xi} T .
\end{aligned}
$$

Because $\Phi(u)$ is bounded, by (20), we can get $\left\{u_{n}\right\}$ is bounded in $E_{0}^{\alpha}$ and $\Phi$ is bounded from below. The proof is completed.

Lemma 18. Assume (H1), (H2) hold, then $\Phi$ satisfies the (PS) condition.

Proof. If $\left\{u_{n}\right\}$ is a (PS) sequence, from Lemma 17, we get $\left\{u_{n}\right\}$ is a bounded sequence in $E_{0}^{\alpha}$. By Lemma 5, we get $\left\{u_{n}\right\}$ has a weakly convergent subsequence. Without loss of generality, we also assume that $u_{n}$ converges weakly to $u_{0}$ in $E_{0}^{\alpha}$, then from (9) and (18), we know

$$
\begin{aligned}
& \left\langle\Phi^{\prime}\left(u_{n}\right)-\Phi^{\prime}\left(u_{0}\right), u_{n}-u_{0}\right\rangle \leq\left\|\Phi^{\prime}\left(u_{n}\right)\right\| \cdot\left\|u_{n}-u_{0}\right\| \\
& \quad-\left\langle\Phi^{\prime}\left(u_{0}\right), u_{n}-u_{0}\right\rangle \longrightarrow 0, \quad n \longrightarrow \infty
\end{aligned}
$$

By Lemma 13 , we can obtain that $u_{n} \longrightarrow u_{0}$ in $C([0, T], R)$, as $n \longrightarrow \infty$, i.e.,

$$
\left\|u_{n}-u_{0}\right\|_{\infty} \longrightarrow 0, \text { as } n \longrightarrow \infty
$$

From (10), we have

$$
\begin{aligned}
& \left\langle\Phi^{\prime}\left(u_{n}\right)-\Phi^{\prime}\left(u_{0}\right), u_{n}-u_{0}\right\rangle \\
& \left.=\int_{0}^{T}-\frac{1}{2}{ }_{0}^{c} D_{t}^{\alpha}\left(u_{n}-u_{0}\right){ }_{t}^{c} D_{T}^{\alpha}\left(u_{n}-u_{0}\right)+{ }_{t}^{c} D_{T}^{\alpha}\left(u_{n}-u_{0}\right){ }_{0}^{c} D_{t}^{\alpha}\left(u_{n}-u_{0}\right)\right] \\
& \quad-\int_{0}^{T}\left(a(t)\left(u_{n}-u_{0}\right)\right)\left(u_{n}-u_{0}\right) d t \\
& \quad-\int_{0}^{T}\left[\nabla F\left(t, u_{n}\right)-\nabla F\left(t, u_{0}\right)\right]\left(u_{n}-u_{0}\right) d t \\
& \quad+\sum_{j=1}^{l}\left[I_{j}\left(u_{n}\left(t_{j}\right)\right)-I_{j}\left(u_{0}\left(t_{j}\right)\right)\right]\left(u_{n}\left(t_{j}\right)-u_{0}\left(t_{j}\right)\right) \\
& \geq \frac{1}{2}|\cos (\pi \alpha)|\left\|u_{n}-u_{0}\right\|_{\alpha}^{2}-\left|\int_{0}^{T}\left[\nabla F\left(t, u_{n}\right)-\nabla F\left(t, u_{0}\right)\right]\right|\left\|u_{n}-u_{0}\right\|_{\infty} \\
& \quad+\left|\sum_{j=1}^{l}\left[I_{j}\left(u_{n}\left(t_{j}\right)\right)-I_{j}\left(u_{0}\left(t_{j}\right)\right)\right]\right|\left\|u_{n}-u_{0}\right\|_{\infty} .
\end{aligned}
$$


By (21), (22), and (23), we can infer that $\left\|u_{n}-u_{0}\right\|_{\alpha}^{2} \longrightarrow 0$, as $n \longrightarrow \infty$, i.e., $u_{n}$ strongly converges to $u_{0}$. Therefore, $\Phi$ satisfies the $(P S)$ condition.

By Lemma 5, we can obtain that there is an orthogonal basis $\left\{e_{i}\right\}$ of $E_{0}^{\alpha}$ such that $E_{0}^{\alpha}=\overline{\operatorname{span}\left\{e_{i}: i=1,2, \cdots\right\}}$. We define $\quad X_{i}:=\operatorname{span}\left\{e_{i}\right\}, V_{k}=\underset{i=1}{\oplus} X_{i}, Y_{k}=\underset{i=k}{\oplus} X_{i}(k=1,2, \cdots)$. Then, $E_{0}^{\alpha}=V_{k} \oplus Y_{k}$.

Proof of Theorem 1. From (H1), (H2), one knows $F(t, 0)=0$ and $I_{j}(0)=0, j=1, \cdots, l$. We find out $\Phi$ has a critical point at 0 . Therefore, we can get the linking $E_{0}^{\alpha}=V_{k} \oplus Y_{k}$ of $\Phi$ at 0 .

According to the equivalence of norm of normed space in finite dimension, there exist positive constants $M_{1}, M_{2}, M_{1}^{\prime}$, $M_{2}^{\prime}$, such that

$$
\begin{gathered}
M_{1}\|u\|_{\alpha} \leq\|u\|_{\infty} \leq M_{2}\|u\|_{\alpha^{\prime}} \\
M_{1}^{\prime}\|u\|_{\alpha} \leq\|u\|_{L^{\nu}} \leq M_{2}^{\prime}\|u\|_{\alpha^{\prime}} \\
u \in V_{k} .
\end{gathered}
$$

First, let $u \in V_{k}$. Because $V_{k}$ is finite dimensional, there exists $0<\rho_{1}<1$ small for $r_{0}>0$, such that

$$
|u(t)| \leq\|u\|_{\infty} \leq M_{2}\|u\|_{\alpha}<M_{2} \rho_{1}<r_{0}, \quad u \in V_{k},\|u\|_{\alpha}<\rho_{1} .
$$

For any $r \in\left(0, r_{0}\right)$, we set $\Omega_{1}=\{t \in[0, T]:|u| \leq r\}, \Omega_{2}=$ $\left\{t \in[0, T]: r \leq|u| \leq r_{0}\right\}, \Omega_{3}=\left\{t \in[0, T]: r_{0} \leq|u|\right\}$, where $[0$, $T]=\cup_{i=1}^{3} \Omega_{i}$ and $\Omega_{i}(i=1,2,3)$ are pairwise disjoint.

Set $F^{*}(t, u)=F(t, u)-C|u|^{\gamma}$, for $\|u\|_{\alpha}<\rho_{1}, u \in V_{k}$, combine $(H 1),(H 3)$ and Lemma 12, we have

$$
\begin{aligned}
\Phi(u)= & \int_{0}^{T}-\frac{1}{2}{ }_{0}^{c} D_{t}^{\alpha} u(t)_{t}^{c} D_{T}^{\alpha} u(t) d t-\frac{1}{2} \int_{0}^{T} a(t) u^{2}(t) d t \\
& -\int_{0}^{T} F(t, u(t)) d t+\sum_{j=1}^{l} \int_{0}^{u\left(t_{j}\right)} I_{j}(s) d s \leq \frac{1}{2|\cos (\pi \alpha)|}\|u\|_{\alpha}^{2} \\
& +\sum_{j=1}^{l} \frac{a_{j}}{\gamma_{j}+1}\left|u\left(t_{j}\right)\right|^{\gamma_{j}+1}-\int_{0}^{T} C|u|^{\gamma} d t-\int_{\Omega_{1}} F^{*}(t, u(t)) d t \\
& -\int_{\Omega_{2}} F^{*}(t, u(t)) d t-\int_{\Omega_{3}} F^{*}(t, u(t)) d t \leq \frac{1}{2|\cos (\pi \alpha)|}\|u\|_{\alpha}^{2} \\
& +\sum_{j=1}^{l} \frac{a_{j} A_{o} \gamma_{j}+1}{\gamma_{j}+1}\|u\|_{\alpha}^{\gamma_{j}+1}-C\|u\|_{L^{\gamma}}^{\gamma}-\int_{\Omega_{1}} F^{*}(t, u(t)) d t \\
& -\int_{\Omega_{2}} F^{*}(t, u(t)) d t-\int_{\Omega_{3}} F^{*}(t, u(t)) d t,
\end{aligned}
$$

where $A_{0}=T^{\alpha-1 / 2} / \Gamma(\alpha) \sqrt{2 \alpha-1}$.

According to (25) and the definition of $\Omega_{3}$ is empty set, we have $\int_{\Omega_{3}} F^{*}(t, u(t)) d t=0$, for any $u \in V_{k}$. By (H3), one has $\int_{\Omega_{2}} F^{*}(t, u(t)) d t \geq C|u|^{\gamma}-C|u|^{\gamma}=0$. On $\Omega_{1}, \quad|u|<r$. From (H3), we can get $\int_{\Omega_{1}} F^{*}(t, u(t)) d t \longrightarrow 0$, as $r \longrightarrow 0$.
Then, $\forall u \in V_{k}, r \in\left(0, r_{0}\right),\|u\|_{\alpha} \leq \rho \leq 1,1<\gamma<\max \left\{\gamma_{j}\right.$ $+1\}<2$, according to (26), we can get

$$
\begin{aligned}
\Phi(u) & \leq \frac{1}{2|\cos (\pi \alpha)|}\|u\|_{\alpha}^{2}+\sum_{j=1}^{l} \frac{a_{j} A_{o}^{\gamma_{j}+1}}{\gamma_{j}+1}\|u\|_{\alpha}^{\gamma_{j}+1}-M_{1}^{\prime \gamma} C\|u\|_{\alpha}^{\gamma} \\
& \leq\|u\|_{\alpha}^{\gamma}\left(\frac{1}{2|\cos (\pi \alpha)|}\|u\|_{\alpha}^{2-\gamma}+\sum_{j=1}^{l} \frac{a_{j} A_{o}^{\gamma_{j}+1}}{\gamma_{j}+1}\|u\|_{\alpha}^{\gamma_{j}+1-\gamma}-M_{1}^{\prime \gamma} C\right) \leq 0 .
\end{aligned}
$$

Hence,

$$
\Phi(u) \leq 0, \quad \forall u \in V_{k},\|u\|_{\alpha} \leq \rho_{1} .
$$

Next, set $u \in Y_{k}$. Because $E_{0}^{\alpha} \longrightarrow C_{0}^{\infty}([0, T, R])$ is continuous compact embedding. Hence, for $u \in Y_{k} \varepsilon>0$, there exists $0<\rho_{2}<1$ small such that $|u| \leq\|u\|_{\infty} \leq T^{2 \alpha-1}\|u\|_{\alpha} / \Gamma^{2}$ $(\alpha)(2 \alpha-1)<T^{2 \alpha-1} \rho_{2} / \Gamma^{2}(\alpha)(2 \alpha-1)<\varepsilon$, for $\|u\|_{\alpha}<\rho_{2}$.

From (H3), $\forall|u|<\varepsilon, u \in Y_{k},\|u\|_{\alpha} \leq \rho_{2}, t \in[0, T]$, there is $\xi \in(0,|\cos (\pi \alpha)|)$, one has

$$
|F(t, u)| \leq(|\cos (\pi \alpha)|-\xi) \frac{\Gamma^{2}(\alpha+1)}{2 T^{2 \alpha}}|u|^{2} .
$$

From (H1), $\forall|u|<\varepsilon, u \in Y_{k},\|u\|_{\alpha} \leq \rho_{2}$, one has

$$
\frac{\left|I_{j}(u)\right|}{|u|^{e_{j}}}>\frac{1}{2} b_{j} .
$$

Let $b=\min _{j=1}^{l} b_{j}, e=\max _{j=1}^{l} e_{j}, \forall|u|<\varepsilon, u \in Y_{k},\|u\|_{\alpha} \leq \rho_{2}<1$, by Lemmas 11 and 12 and (29) and (30), we obtain

$$
\begin{aligned}
\Phi(u)= & \int_{0}^{T}-\frac{1}{2}{ }_{0}^{c} D_{t}^{\alpha} u(t){ }_{t}^{c} D_{T}^{\alpha} u(t) d t-\frac{1}{2} \int_{0}^{T} a(t) u^{2}(t) d t \\
& -\int_{0}^{T} F(t, u(t)) d t+\sum_{j=1}^{l} \int_{0}^{u\left(t_{j}\right)} I_{j}(s) d s \geq \frac{1}{2}|\cos (\pi \alpha)|\|u\|_{\alpha}^{2} \\
& -(|\cos (\pi \alpha)|-\xi) \frac{\Gamma^{2}(\alpha+1)}{2 T^{2 \alpha}}\|u\|_{L^{2}}^{2}-\frac{1}{2} a_{1} T|u|^{2}+\frac{1}{2} \frac{b l}{e+1}|u|^{e+1} \\
\geq & \frac{1}{2}|\cos (\pi \alpha)|\|u\|_{\alpha}^{2}-\frac{1}{2}(|\cos (\pi \alpha)|-\xi)\|u\|_{\alpha}^{2}-\frac{1}{2} a_{1} T|u|^{2} \\
& +\frac{1}{2} \frac{b l}{e+1}|u|^{e+1} \geq \frac{1}{2}\left(\xi\|u\|_{\alpha}^{2}+|u|^{e+1}\left(\frac{b l}{e+1}-a_{1} T|u|^{1-e}\right)\right)>0 .
\end{aligned}
$$

Hence,

$$
\Phi(u)>0, \forall u \in Y_{k},\|u\|_{\alpha} \leq \rho_{2} .
$$

Let $\rho=\min \left\{\rho_{1}, \rho_{2}\right\}$, from (28) and (32), we obtain

$$
\begin{aligned}
& \Phi(u) \leq 0, \quad \forall u \in V_{k},\|u\|_{\alpha} \leq \rho, \\
& \Phi(u)>0, \quad \forall u \in Y_{k},\|u\|_{\alpha} \leq \rho .
\end{aligned}
$$

It follows from Lemmas 17 and 18 that $\Phi$ is bounded from below and satisfies the $(P S)$ condition. Then, from Lemma 15, 
we can get $\Phi$ has at least two nonzero critical points, and $C_{k}$ $(\Phi, 0) \cong 0$, so $u=0$ is a homological nontrivial point of $\Phi$. Hence, the system (1) has at least three classical solutions.

Proof of Theorem 2. According to (H4), we can deduce that $\Phi$ is even. By Lemmas 17 and 18, we know $\Phi$ is bounded from below and satisfies the (PS) condition. For given $\rho>0$, set $E^{\prime}=S_{p}=\left\{u \in V_{k}:\|u\|=\rho\right\}$. By (27), if $\rho$ is small enough, one has $\sup _{E^{\prime}} \Phi(u)<0$. Clearly, $\operatorname{dim} V_{k}=k$. Then, we can conclude that $\Phi$ has at least $k$ distinct pairs of critical points from Lemma 16. Hence, the system (1) has at least $k$ distinct pairs of classical solutions. We complete the proof.

Example 19.

$$
\left\{\begin{array}{l}
-\frac{1}{2} \frac{d}{d t}\left({ }_{0} D_{t}^{-0.6}+{ }_{t} D_{T}^{-0.6}\right) u^{\prime}(t)=\frac{2}{5} u(t)+\nabla F(t, u(t)), \quad t \neq t_{j}, \text { a.e. } t \in[0,1] \\
\Delta\left(D_{t}^{0.7} u\right)\left(t_{j}\right)=I_{j}\left(u\left(t_{j}\right)\right), \quad t_{j} \in[0,1], j=1,2, \cdots, l, \\
u(0)=u(T)=0 .
\end{array}\right.
$$

According to (34), we can see that $\beta=0.6, \alpha=0.7, a(t)$ $=2 / 5, T=1$.

Let $I_{j}(u)=(4 / 3) u^{1 / 3}$, then the condition $(H 1)$ holds with $a_{j}=3 / 2, \gamma_{j}=1 / 3, b_{j}=4 / 3, e_{j}=1 / 3$.

Let $F(t, u(t))=1 / 15\left(1+\sin ^{2} t\right)|u|^{2}\left(1 / \ln \left(|u|^{2 / 3}+1.5\right)\right)$, $a_{1}=9 / 20$. By simple calculations, we can get $\Gamma^{2}(\alpha+1) / T^{2 \alpha}$ $\approx 0.84474,|\cos (0.7 \pi)| \approx 0.58778$,

$\lim _{|u| \rightarrow \infty} \sup \frac{F(t, u)}{|u|^{2}} \longrightarrow 0<\frac{1}{2}\left(\frac{\Gamma^{2}(\alpha+1)}{T^{2 \alpha}}|\cos (\pi \alpha)|-a_{1}\right) \approx 0.02326 ;$

then, the condition ( $H 2)$ holds.

By $(H 3)$, we know

$$
\lim _{|u| \rightarrow 0} \sup \frac{|F(t, u)|}{|u|^{2}} \leq 0.32884<\frac{\Gamma^{2}(\alpha+1)}{2 T^{2 \alpha}} \approx 0.42237
$$

Let $r=e-1.7, r_{0}=e-1.5, C=2 / 25, \gamma=6 / 5$; then, for $e-1.7 \leq|u| \leq e-1.5$, we have

$$
F(t, u)>0.14471|u|^{2} \geq \frac{2}{25}|u|^{6 / 5}
$$

then, the condition $(H 3)$ is satisfied.

It easy to see that the condition (H4) holds.

According to Theorem 1, the system (1) exists at least three classical solutions. According to Theorem 2, the system (1) possesses at least $k$ distinct pairs of classical solutions.

\section{Conclusions}

In this work, we study perturbed fractional differential equation with impulses. We give sufficient conditions of the existence of at least three classical solutions and at least $k$ distinct pairs of classical solutions for problems (1), where $k$ is the dimension of $V_{k}$.

\section{Data Availability}

No data were used to support this study.

\section{Conflicts of Interest}

The authors declare no conflict of interest.

\section{Authors' Contributions}

The authors declare that the study was realized in collaboration with the same responsibility. All authors read and approved the final manuscript.

\section{Acknowledgments}

The research is supported by the National Natural Science Foundation of China (No. 61673008) and the Young Backbone Teacher Funding Scheme of Henan (No.2019GGJS079).

\section{References}

[1] V. J. Ervin and J. P. Roop, "Variational formulation for the stationary fractional advection dispersion equation," Numerical Methods for Partial Differential Equations, vol. 22, no. 3, pp. 558-576, 2006.

[2] A. A. Kilbas, H. M. Srivastava, and J. J. Trujillo, "Theory and Applications of Fractional Differential Equations," in NorthHolland Mathematics studies, p. 204, Elsevier, Amsterdam, 2006.

[3] V. Lakshmikantham, S. Leela, and J. V. Devi, Theory of Fractional Dynamic Systems, Camb. Sci. Publ, Cambridge, 2009.

[4] I. Podlubny, Fractional Differential Equations, Academic Press, New York, 1999.

[5] J. N. Corvellec, V. V. Motreanu, and C. Saccon, "Doubly resonant semilinear elliptic problems via nonsmooth critical point theory," Journal of Differential Equations, vol. 248, no. 8, pp. 2064-2091, 2010.

[6] Z. Hu, W. Liu, and J. Liu, "Ground state solutions for a class of fractional differential equations with Dirichlet boundary value condition," Abstract and Applied Analysis, vol. 2014, Article ID 958420, 7 pages, 2014.

[7] Y. Li, H. Sun, and Q. Zhang, "Existence of solutions to fractional boundary-value problems with a parameter," Electronic Journal of Differential Equations, vol. 2013, pp. 1-12, 2013.

[8] F. Jiao and Y. Zhou, "Existence of solutions for a class of fractional boundary value problems via critical point theory," Computers \& Mathematcs with Applications, vol. 62, no. 3, pp. 1181-1199, 2011.

[9] F. Jiao and Y. Zhou, "Existence results for fractional boundary value problem via critical point theory," International Journal of Bifurcation and Chaos, vol. 22, no. 4, article 1250086, 2012.

[10] J. Chen and X. H. Tang, "Existence and multiplicity of solutions for some fractional boundary value problem via critical point theory," Abstract and Applied Analysis, vol. 2012, Article ID 648635, 21 pages, 2012. 
[11] R. Rodríguez-López and S. Tersian, "Multiple solutions to boundary value problem for impulsive fractional differential equations," Fractional Calculus and Applied Analysis, vol. 17, no. 4, pp. 1016-1038, 2014

[12] P. Li, C. Xu, and H. Wang, "Weak solutions to boundary value problems for fractional differential equations via variational methods," Journal of Nonlinear Sciences and Applications, vol. 9, no. 5, pp. 2971-2981, 2016.

[13] X. B. Shu, Y. Lai, and Y. Chen, "The existence of mild solutions for impulsive fractional partial differential equations," Nonlinear Analysis, vol. 74, no. 5, pp. 2003-2011, 2011.

[14] P. Li and C. Xu, "Boundary value problems of fractional order differential equation with integral boundary conditions and not instantaneous impulses," Journal of Function Spaces, vol. 2015, Article ID 954925, 9 pages, 2015.

[15] N. Nyamoradi and R. Rodríguez-López, "On boundary value problems for impulsive fractional differential equations," Applied Mathematics and Computation, vol. 271, pp. 874892, 2015.

[16] Y. Guo, X. B. Shu, Y. Li, and F. Xu, "The existence and HyersUlam stability of solution for an impulsive Riemann-Liouville fractional neutral functional stochastic differential equation with infinite delay of order $1<\beta<2$," Boundary Value Problems, vol. 2019, no. 1, Article ID 59, 2019.

[17] X. B. Shu, Y. Lai, and F. Xu, "Existence of subharmonic periodic solutions to a class of second-order non-autonomous neutral functional differential equations," Abstract and Applied Analysis, vol. 2012, Article ID 404928, 26 pages, 2012.

[18] X. Shu, Y. Lai, and F. Xu, "Existence of infinitely many periodic subharmonic solutions for nonlinear non-autonomous neutral differential equations," Electronic Journal of Differential Equations, vol. 2013, no. 150, pp. 1-21, 2013.

[19] P. Li, L. Xu, H. Wang, and Y. Wang, "The existence of solutions for perturbed fractional differential equations with impulses via Morse theory," Boundary Value Problems, vol. 2020, no. 1, Article ID 21, 2020.

[20] Y. Zhao, H. Chen, and C. Xu, "Nontrivial solutions for impulsive fractional differential equations via Morse theory," Applied Mathematics and Computation, vol. 307, pp. 170179, 2017.

[21] R. P. Agarwal, T. G. Bhaskar, and K. Perera, "Some results for impulsive problems via Morse theory," Journal of Mathematical Analysis and Applications, vol. 409, no. 2, pp. 752-759, 2014.

[22] K. Ait-Mahiout, S. Djebali, and T. Moussaoui, "Multiple solutions for an impulsive boundary value problems on the halfline via Morse theory," Topological Methods in Nonlinear Analysis, vol. 47, no. 1, pp. 219-240, 2015.

[23] Y. Zhao, X. Shi, and H. Chen, "Multiplicity results for a class of fractional differential equations with impulse," Advances in Difference Equations, vol. 2018, no. 1, Article ID 341, 2018.

[24] J. Liu and J. Su, "Remarks on multiple nontrivial solutions for quasi-linear resonant problems," Journal of Mathematical Analysis and Applications, vol. 258, no. 1, pp. 209-222, 2001.

[25] P. Li, J. Ma, H. Wang, and Z. Li, "Infinitely many nontrivial solutions for fractional boundary value problems with impulses and perturbation," The Journal of Nonlinear Sciences and Applications, vol. 10, no. 5, pp. 2283-2295, 2017.
[26] H. Brezis and L. Nirenberg, "Remarks on finding critical points," Communications on Pure and Applied Mathematics, vol. 44, no. 8-9, pp. 939-963, 1991.

[27] P. H. Rabinowitz, "Minimax methods in critical point theory with applications to differential equations," in CBMS Reg.Conf. Ser. Math, vol. 65, American Mathematical Society, Providence, 1986. 